\section{Precurrence risk of neural tube defects in siblings of infants with lipomyelomeningocele}

\section{To the Editor:}

We read with interest the recent article that found the recurrence risk of any type of neural tube defect (NTD) among siblings of individuals with lipomyelomeningocele to be consistent with that for individuals with open neural tube defects. ${ }^{1}$

The Hawaii Birth Defects Program (HBDP) is an active, population-based birth defects registry for the entire state of Hawaii. Among the information the HBDP collects on each case are the total number of older siblings (including those not born alive) and family history of birth defects. The HBDP identifies information on the family history of birth defects through review of the mother's prior pregnancy history and the genetic counseling information in medical records as well as through linkage of cases within the HBDP database. ${ }^{2}$ From this information the precurrence risk (risk among older siblings) for selected birth defects can be determined.

Among deliveries during 1986 through 2002, the HBDP identified 22 cases with lipomyelomeningocele and 294 cases with other NTDs (anencephaly, spina bifida, and encephalocele). The number of older siblings was known for 292 of the other NTD cases, which had a total of 516 older siblings. Six of these siblings had a NTD, none of which were lipomyelomeningocele. Thus the precurrence risk for NTDs among siblings of infants with other NTDs was $1.16 \%$. The number of older siblings was known for 20 of the lipomyelomeningocele cases, which had a total of 32 older siblings. One of these siblings had an NTD, which was spina bifida. Thus, the precurrence risk among siblings of infants with lipomyelomeningocele was $3.13 \%$, which was not significantly different from the precurrence risk among siblings of infants with other NTDs (rate ratio $2.69,95 \%$ confidence interval $0.06-22.15$ ). For both the lipomyelomeningocele and other NTD cases, no family members other than siblings were reported to have had NTDs.

Like the previous investigation, the analysis of the HBDP data is limited by the small sample size. However, the $3 \%$ precurrence risk of NTDs in siblings of infants with lipomyelomeningocele was similar to the $4 \%$ recurrence risk reported by the prior study. Moreover, the precurrence risk of NTDs in siblings of infants with lipomyelomeningocele did not differ significantly from that for infants with other NTDs, and both were similar to the $2 \%$ to $5 \%$ recurrence risk reported in the literature. Despite its limitations, the HBDP data would tend to support the suggestion in the previous investigation that the underlying genetic factors for lipomyelomeningocele and open NTDs may be similar.

\section{Acknowledgments}

Funding was provided by the Hawaii State Department of Health, Children With Special Health Needs Branch, Centers for Disease Control and Prevention, Ronald McDonald Childrens' Charities, March of Dimes Birth Defects Foundation, George F. Straub Trust, Queen Emma Foundation, Pacific Southwest Regional Genetics Network, and Kamehameha Schools/Bishop Estate.

\section{References}

1. Sebold CD, Melvin EC, Siegel D, Mehltretter L, Enterline DS, Nye JS et al; NTD Collaborative Group. Recurrence risks for neural tube defects in siblings of patients with lipomyelomeningocele. Genet Med 2005;7:64-67.

2. Forrester MB, Merz RD. Identification of family history of birth defects by a birth defects registry. J Registry Management 2002;29:9-14.

Mathias B. Forrester, BS

Ruth D. Merz, MS

Hawaii Birth Defects Program Honolulu, Hawai 